\title{
Mechanical Behaviour of 3D Printed PLA Hollow Spherical Parts Under Axial Compression
}

\author{
ADELINA HRITUC ${ }^{1}$, ANDREI MIHALACHE ${ }^{1}$, MARIAN MARES ${ }^{2}$, \\ MARGARETA COTEATA ${ }^{1}$, OANA DODUN ${ }^{1 *}$, GHEORGHE NAGIT ${ }^{1}$, \\ LAURENTIU SLATINEANU ${ }^{1}$ \\ ${ }^{1}$ Gheorghe Asachi Technical University of Iasi, Department of Machine Manufacturing Technology, Blvd. D. Mangeron, \\ 59A, 700050, Iasi, Romania \\ ${ }^{2}$ Gheorghe Asachi Technical University of Iasi, Department of Mechanical Engineering, Mechatronics and Robotics, 59A D. \\ Mangeron, Blvd., 700050, Iasi, România
}

\begin{abstract}
The form of the outer and inner surfaces of hollow spherical parts determines the developments of some particular categories of efforts during the compression tests. The overall purpose of the research presented in this paper was to study the behaviour of the hollow spherical parts under axial compression. The PLA hollow spherical parts were obtained by $3 D$ printing and using distinct values for certain process input factors. The finite element method was used to theoretically investigate the behaviour of the parts and it highlighted the total plastic deformation of the test pieces. To experimentally verify the theoretical considerations, an L9 Taguchi orthogonal design was performed. The empirical mathematical model thus determined highlighted the stronger influence exerted by the printing plate temperature, printing speed, and part wall thickness.
\end{abstract}

Keywords: Cave spherical part, Plastic material, 3D printing, Compression stress, Experimental tests, Mathematical model.

\section{Introduction}

The spherical parts can be made of different types of metallic materials, which are used as components of the bearings, for example, to ensure the movement of other components. Also, it can be used in spherical joints, allowing rotations around 3 mutually perpendicular directions. Generally, due to the constructive shape, the spherical surfaces ensure smooth sliding of one part towards the other, being the intermediate component in various assemblies.

In practice, full spherical or cave/hollow spherical parts are used. The advantages and disadvantages are specific to the considered situation. The filled spheres are more rigid and seem easier to technologically be obtained, while cave spheres can be more elastic, requiring less consumption of material. As used materials, metallic and non-metallic materials may be considered, with plastics presenting a particular interest.

The cave parts have a hollow core inside, according to the chosen application. The spherical cave pieces can be obtained by casting, injection, roto-molding or additive manufacturing processes. Among this large variety of manufacturing methods, the process of manufacturing the parts by adding material appears to be quite interesting in terms of the parts mechanical properties, surface roughness, surface layer properties, material homogeneity and specific properties generated by the combination of the distinct processes with specific materials.

As a result of obtaining spherical cave pieces, some problems could be formulated about their behavior under certain mechanical stresses. Certain research aspects concerning the behavior of the spherical parts under the action of certain mechanical efforts were investigated by the researchers.

Thus, Yuan et al. have created a structure composed of spherical shells obtained by a 3D printing process (selective laser sintering). They observed the distinct behavior of the hollow spherical blocks affected by a compression process [1]. Such structures have led to the generation of auxetic metamaterials characterized by high energy absorption.

\footnotetext{
*email:oanad@tcm.tuiasi.ro
} 
Structures that included hollow spherical components were developed by Kohnen et al. using a 3D printing method [2]. Selective laser melting has been used to generate lattice structures that have proven interesting properties from compression behavior and also a high energy absorption capacity.

Yazifar et al. considered a 3D printing process applied to generate hollow spherical structures whose behavior during compression testing was investigated [3]. They observed that the reduction of Young's modulus and stress depends on the volume of the spheres.

M. Koopman et al. have studied the behavior of the microspheres under uniaxial compression efforts [4]. The result of the research showed that there is a dependence between the diameter and the load to failure and also between the sphere diameter and fracture energy.

Research concerning the use of the finite element method to simulate the behavior of the hollow sphere structures under uniaxial compression was developed by Amani and Öchsner [5]. They observed an exponential growth of Young's modulus when the average density increases for different base materials.

The research whose results are presented in this paper aimed to highlight the behavior of cave spheres made of plastic under compression stress. Certain manufacturing process conditions were considered as input factors in experimental research.

\section{Theoretical considerations}

Any shape or surface can be defined by a mathematical equation. In the case of the spherical surface, the equation is:

$$
\left(x-x_{0}\right)^{2}+\left(y-y_{0}\right)^{2}+\left(z-z_{0}\right)^{2}=r^{2},
$$

where $x, y, z$, are the coordinates of a point found on the spherical surface, $x_{0}, y_{0}, z_{0}$ are constants on the cartesian directions and $r$ is the radius of the sphere.

Let $a, b, c, d, e$ be real numbers with $a \neq 0$ and:

$$
x_{0}=\frac{-b}{a}, y_{0}=\frac{-c}{a}, x_{0}=\frac{-d}{a}, \rho=\frac{b^{2}+c^{2}+d^{2}+a e}{a^{2}} .
$$

In such a case, the following relation could be written:

$$
f(x, y, z)=a\left(x^{2}+y^{2}+z^{2}\right)+2(b x+c y+d z)+e=\mathbf{0} .
$$

The above-mentioned relation can be considered as a general equation for the sphere description.

In the case of the technological process of manufacturing the spherical parts, some process input factors can influence the surface roughness of the final product. If 3D printing is taken into consideration as a manufacturing solution, then the process seems to be simple enough and of good quality from the surface aspect.

In practice, there is some important kind of stresses which are convenient for the technical uses of the parts. For example, the compression stress reveals how resistant is a spherical part under a certain force until the part is deformed and loses the properties of being used.

Thus, the research idea was to obtain some spherical hollow part by printing and test them to know what resistance they have under compression stress, what is their elastic behavior and other mechanical properties which recommend the use of these parts. Sometimes there are situations when the elastic behavior under compression axial forces is of technical and functional interest.

It is expected that under the axial compression effort, an initial spherical part (Figure 1) will be elastically and plastically deformed and this situation could lead to a change of its initial shape (Figure 2). Analyzing the behavior of other materials and comparing their properties, our initial hypothesis about the result of the tests was that at a maximum loading of the compression force, the hollow spherical part will crack and break into two halves. A schematic representation of this situation can be seen in Figure 2.

As a piece of initial information for performing the compression tests, the dimensions of the test piece, kind of used plastic material, 3D printing equipment with the necessary setup of the manufacturing 
conditions can be taken into account. All of these aspects need the considering of some factors that can influence the mechanical properties of the printed parts. Taking into consideration some of the part characteristics and manufacturing process input factors, the decision to use an L9 Taguchi orthogonal design, with 4 independent variables at 3 experimental levels was formulated. Among all the possible combinations of the four factors, the L9 Taguchi orthogonal design takes into consideration only 9 combinations, whose results are able to highlight accurately enough the effect exerted by the testing input factors on the elastic behavior of the plastic spherical hollow parts under the action of axial compression efforts.

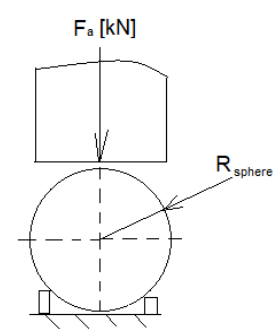

Figure 1. Initial conditions of testing the spherical parts under compression efforts

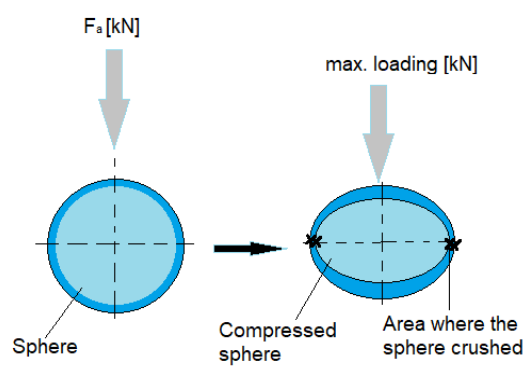

Figure 2. Expected elastic behavior of a spherical hollow part under the action of an axial compression force $F_{a}$

In order to better characterize the theoretical behavior of the plastic spherical hollow parts under the action of the axial compression efforts, a simulation utilizing the finite element method was developed. Generally, this method offers the possibility to formulate some remarks about the material behavior before developing the experimental research and it could be used inclusively to define the values of the process input factors. Subsequently, the method could be used to compare its results with the results experimentally obtained. The analysis allows also a certain prediction concerning the risk of crack generation.
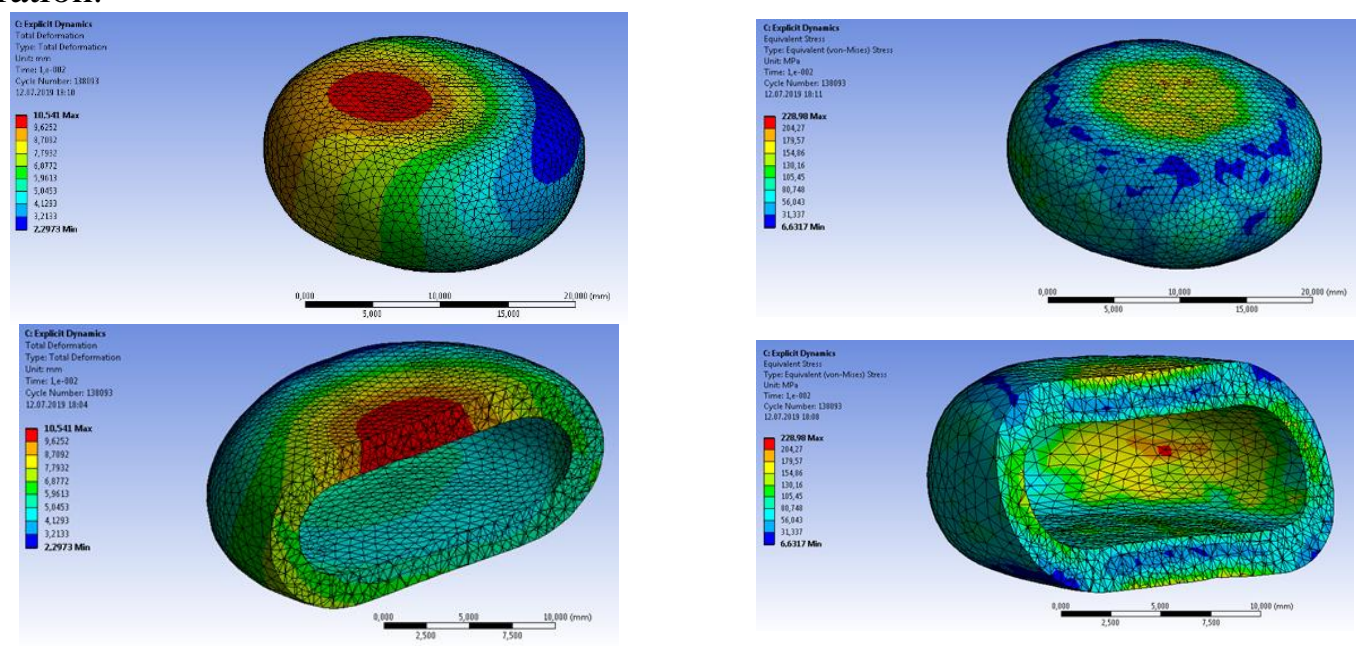

Figure 3. Simulation using the finite element method of the new shape of the plastic spherical hollow part under the action of the axial compression force (wall thickness $\mathrm{h}=2 \mathrm{~mm}$, axial force max. $\mathrm{Fa}=7.189,97 \mathrm{daN}$ )

As a test sample material, the polylactide (PLA) was used, taking into account the information found in the Ansys's database for a polyethylene type nonlinear material. The values of $1100 \mathrm{MPa}$ for the Young modulus, 0.42 for the Poisson ratio and $25 \mathrm{MPa}$ for the tensile yield strength are specific to this material. The meshing procedure was based on explicit dynamics, like fractures or large deformations are expected. The mesh included 58650 elements and 20139 nodes. An adaptive sizing approach with a smooth transition set for inflation was used. The printing plate was considered as fixed support. A gradually ramped displacement in ten timestamps along the $Z$-axis for the deformation element was 
considered for more than half of the test piece height. The analysis was stopped to not exceed a geometric strain of $150 \%$ or when the material failure occurs. The maximum energy error was set at $1 \%$.

Taking into consideration the mechanical properties of the spherical hollow part material, its dimensions and certain values of the axial force, some information concerning the behavior of the parts were obtained (Figure 3). As computer simulation software, the ANSYS R19.2 software was used. The simulation was based on the values specified for compression stresses, considering the material and wall thickness of the sphere and the result showed that in the imposed investigation conditions, the main stresses appear initially in the superior and inferior zone of the test piece (Figure 3). The simulation images highlighted the total deformation of the test piece under the action of a pre-established value of the axial compression force, considering inclusively the equivalent-stresses (von Misses). A crosssection of the spherical hollow part showed the part internal shape at the end of the proposed plastic deformation process.

\section{Design and performing of the experimental tests}

The experimental tests were designed to investigate the behavior of the plastic hollow spherical parts made by 3D printing under axial compression (fig. 4) and considering some geometrical characteristics and manufacturing conditions as testing process input factors. The spherical hollow parts were not additionally finished after the 3D printing process, to not introduce elements that could change their behavior under the axial compression.

The test piece material was a silver metallic Ultimaker PLA, with a mass distribution of $750 \mathrm{mg}$ for $2.85 \mathrm{~mm}$. The recommended melting temperature for this material is in the range of $195-240{ }^{\circ} \mathrm{C}$, while the heated plate temperature was set to tape or $64^{\circ} \mathrm{C}$. The diameter of the nozzle orifice was of $0.4 \mathrm{~mm}$, while the layer thickness was of $0.1 \mathrm{~mm}$.

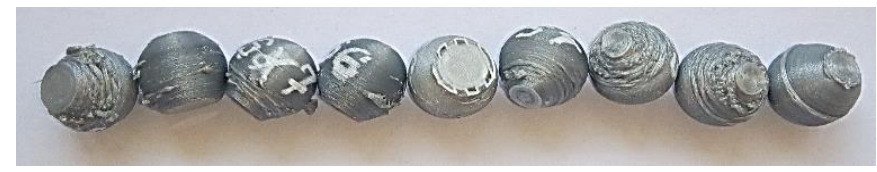

Figure 4. Test pieces obtained by $3 \mathrm{D}$ printing before applying the axial compression tests

As initial information for the compression tests, the dimensions of the test piece, the type of plastic material used, the 3D printing equipment with the necessary setup of the manufacturing conditions were taken into account. All of these factors need the considering of some factors that can influence the mechanical properties of the printed parts. With this aim in view, the L9 Taguchi orthogonal design with 4 independent variables at 3 experimental levels was used [4].

As above-mentioned, the hollow plastic spherical parts were achieved using a 3D printing equipment and the PLA as a material to be deposited during the process of parts generation. Within the manufacturing process of the spherical hollow parts, it was considered that some of the values of the 3D printing process parameters could be established by the operator or by the designer of the process and the problem if the values of these parameters could influence the behavior of the parts under the axial compression tests was formulated. During the printing process, a disturbing factor, the ambient temperature, seems to have a considerable influence on the outer quality of the parts. That is why, even though there where some preliminary pieces in order to stabilize the input factors for better quality, the test pieces have the aspect as in Figure 4.

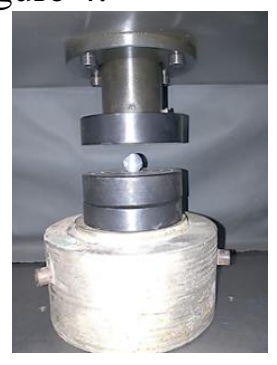

Figure 5. Position of the plastic spherical hollow part on the testing equipment before applying the axial compression force 
As 3D printing process input factors, the wall thickness $h$ of spherical hollow part, the printing speed $v$, the cooling level $c$, and the temperature $\theta$ of the plate on which the spherical hollow part was gradually generated were selected. Appreciating that for practical applications, the elastic behavior of the plastic spherical hollow parts under axial compression efforts could be of technical and scientific interest, the elasticity limit for the parts obtained in distinct manufacturing conditions were considered as a manufacturing process output parameter. The assumption that between the limits of the process input factors variation, a possible not monotone variation of the process output parameter could be valid was formulated and in this case, the tests could be performed for three levels of the process input factors variation.

After obtaining the spherical hollow parts, the tests were made using universal testing equipment whose work zone could be seen in figure 5. Because of the spherical shape of the part, manufacturing and also a testing problem was the positioning and keeping the piece in a fix position. With this aim in view a small dimension flat part that facilitated the contact between the part and the printing plate, respectively the positioning of the part during the axial compression test was used.

The structure of the L9 Taguchi orthogonal design could be seen in table 1 .

Table 1. Experimental conditions and results

\begin{tabular}{|c|c|c|c|c|c|}
\hline \multirow{4}{*}{$\begin{array}{l}\text { Values of the } \\
\text { process input } \\
\text { factors }\end{array}$} & \multicolumn{5}{|c|}{ Wall thickness, $h: 2 ; 4 ; 6 \mathrm{~mm}$} \\
\hline & \multicolumn{5}{|c|}{ Printing speed, $v: 50 ; 75 ; 100 \mathrm{~mm} / \mathrm{s}$} \\
\hline & \multicolumn{5}{|c|}{ Cooling conditions, $c: 0 ; 50 ; 100 \%$} \\
\hline & \multicolumn{5}{|c|}{ Plate temperature $\theta: 45 ; 60 ; 75 \circ \mathrm{C}$} \\
\hline \multicolumn{6}{|c|}{ Experimental conditions and results for each test } \\
\hline Experiment & \multicolumn{4}{|c|}{ Manufacturing process input factors mentioned by codded values } & Testing process output \\
\hline & $\begin{array}{c}\text { Wall } \\
\text { thickness } h \text {, } \\
\text { mm }\end{array}$ & $\begin{array}{c}\text { Printing } \\
\text { speed } v, \mathrm{~mm} / \mathrm{s}\end{array}$ & $\begin{array}{c}\text { Cooling } \\
\text { conditions, } c, \\
\%\end{array}$ & $\begin{array}{c}\text { Plate } \\
\text { temperature, } \\
\theta,{ }^{\circ} \mathrm{C}\end{array}$ & $\begin{array}{l}\text { Maximum load for elasticity } \\
\text { zone, } F, \mathrm{kN}\end{array}$ \\
\hline 1 & 2 & 50 & 0 & 45 & 3.083 \\
\hline 2 & 2 & 75 & 50 & 60 & 1.05 \\
\hline 3 & 2 & 100 & 100 & 75 & 1 \\
\hline 4 & 4 & 50 & 50 & 75 & 0.81 \\
\hline 5 & 4 & 75 & 100 & 45 & 2.97 \\
\hline 6 & 4 & 100 & 0 & 60 & 7.39 \\
\hline 7 & 6 & 50 & 100 & 60 & 2.7 \\
\hline 8 & 6 & 75 & 0 & 75 & 4.2 \\
\hline 9 & 6 & 100 & 50 & 45 & 5.4 \\
\hline
\end{tabular}

The 3D printing equipment type Ultimaker $2+$ (made in the Netherlands) was used to obtain the plastic spherical hollow parts by the above-mentioned manufacturing conditions.

The testing of the plastic spherical hollow parts to the compression efforts was performed on a computer-controlled electronic universal testing machine (WDW type, made in China). The cross-head speed was of $1 \mathrm{~mm} / \mathrm{min}$. An example of an experimental load-deformation curve obtained using the universal testing machine operating software is presented in Figure 6.

A global examination of this graphical representation shows that there is a zone of elastic behavior, followed by a curvilinear zone that corresponds to a plastic behavior, a horizontal linear zone when there is a continuous deformation without an increase of the deformation force and afterward an increase of the deformation as the load increases, too (Figure 6). The experimental tests were stopped when the parts deformations exceeded generally $7 \mathrm{~mm}$.

As a consequence of applying the compression load and due to the plasticity of the parts material, after exceeding the elasticity limits during the experimental tests, the deformed parts presented two parallel flat surfaces that correspond to the small flat surface generated on the base plate during the 3D printing process and the second flat surface generated by the surface of the testing machine moving subassembly. 


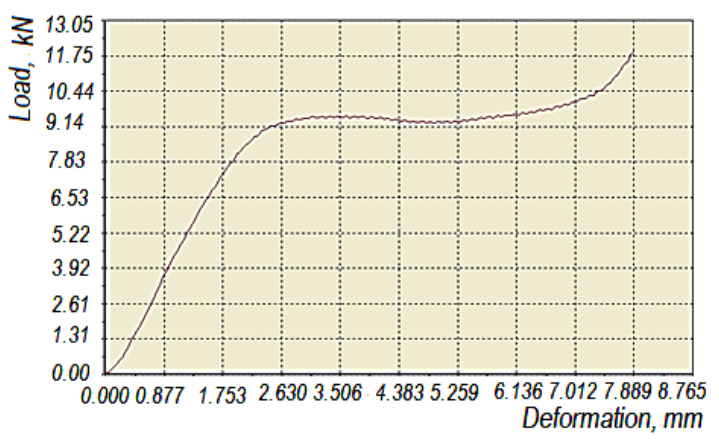

Figure 6. Example of load-deformation diagram obtained by testing a plastic spherical hollow part at compression efforts (experiment no. 2: part thickness $\mathrm{h}=2 \mathrm{~mm}$, printing speed $\mathrm{v}=75 \mathrm{~mm} / \mathrm{s}$, cooling level $\mathrm{c}=50 \%$, plate temperature $\theta=60^{\circ} \mathrm{C}$ )
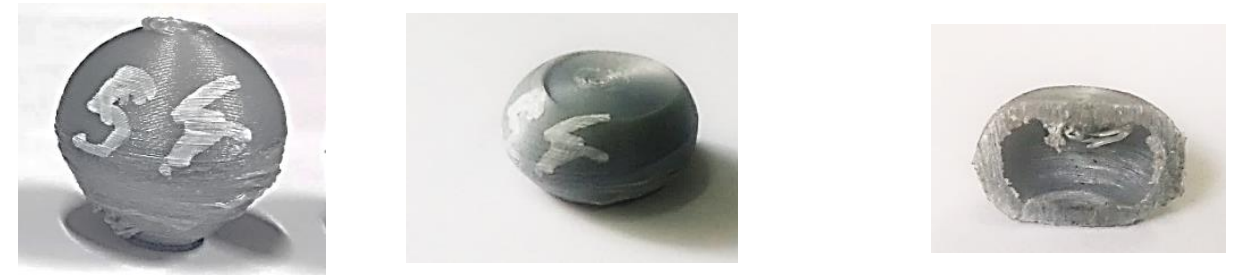

Figure 7. The aspect of the initial part shape, of the deformed part after the application of the compression test and the internal surface that corresponds to the deformed part (initial part thickness $h=2 \mathrm{~mm}$, printing speed $v=75 \mathrm{~mm} / \mathrm{s}$, cooling level $c=50 \%$, plate temperature $\theta=65^{\circ} \mathrm{C}$ )

In Figure 7, one could observe the aspect of the initial hollow spherical part, of the part shape after the applying of the compression test and a section through the deformed part, to highlight the shape of the part internal surface.

\section{Mathematical modelling of the experimental results}

The experimental results were mathematically modeled utilizing specialized software that uses the method of least square to identify an empirical mathematical model by taking into consideration the experimental results [5]. The software allows highlighting the most adequate empirical mathematical model among five such models (polynomial of the first and second degree, power type, exponential type, hyperbolic type). As a criterion of evaluating the adequacy of a certain mathematical empirical model to the considered experimental results, the so-called Gauss's criterion is used. The Gauss's criterion value is determined as a sum of the squares of differences between the ordinates that correspond to the considered empirical mathematical model and the ordinates that correspond to the experimental results, for the same values of the input factors.

Using the above-mentioned specialized software, the following empirical mathematical model was obtained for the maximum force that generates the elastic deformation of the spherical hollow part:

$$
F=12.465 h^{0.919} v^{0.884} c^{-0.0543} \theta^{-1.635}
$$

On the base of the above-mentioned empirical mathematical model, the graphical representations from figures 8 and 9 were elaborated.

The analysis of this empirical mathematical model and the graphical representations from Figures 8 and 9 facilitated the formulation of some general remarks. Thus, one notice that the most important factor able to affect the plastic spherical hollow parts under axial compression test is the plate temperature, whose exponent in the empirical mathematical model has the highest absolute value in comparison with the values of the exponents attached to the other manufacturing process input factors. One could explain this fact by the lower connection among the deposited layers due to the increase of the printing plate temperature. 


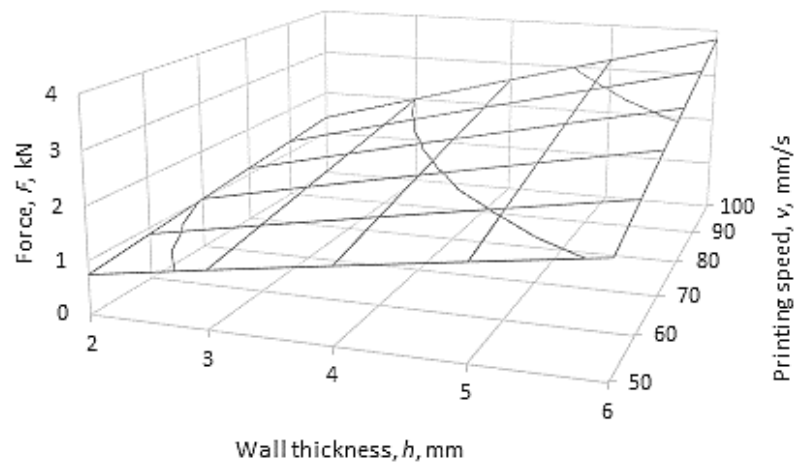

Figure 8. Effect of part wall thickness and the printing speed on the maximum force in the elasticity zone

$$
\left(c=50 \%, \theta=60{ }^{\circ} \mathrm{C}\right)
$$

As expected, the increase of the wall thickness determined an increase of the spherical hollow parts elasticity.

The very low value of the exponent attached to the cooling conditions factor $(-0.0543)$ showed that practically this process input factor does not exert influence on the spherical hollow part elasticity for the considered experimental conditions.

An approximately equal effect on the elastic behavior of the spherical hollow parts is exerted by the wall thickness and printing speed since in the empirical mathematical model similar subunit values are attached to these independent variables. The increase of both the wall thickness and printing speed has, as a result, an increase of the elasticity of the spherical parts since the exponents' values are positive.

One could remark the similarities between the plastic spherical hollow parts behaviors simulated employing the finite element method and the real behavior proved by the experimental testing; this means that the considerations formulated when theoretically analyzing the deformation conditions of the spherical parts were correct. For example, using $\mathrm{h}=2 \mathrm{~mm}$, the force able to determine plastic deformation of about $10 \mathrm{~mm}$ evaluated using finite element analysis was of about $7.189 \mathrm{daN}$, while using the empirical mathematical model $\left(h=2 \mathrm{~mm}, v=100 \mathrm{~mm} / \mathrm{s}, c=0 \%\right.$, and $\left.\theta=23^{\circ} \mathrm{C}\right)$ the force was of $7.515 \mathrm{daN}$.

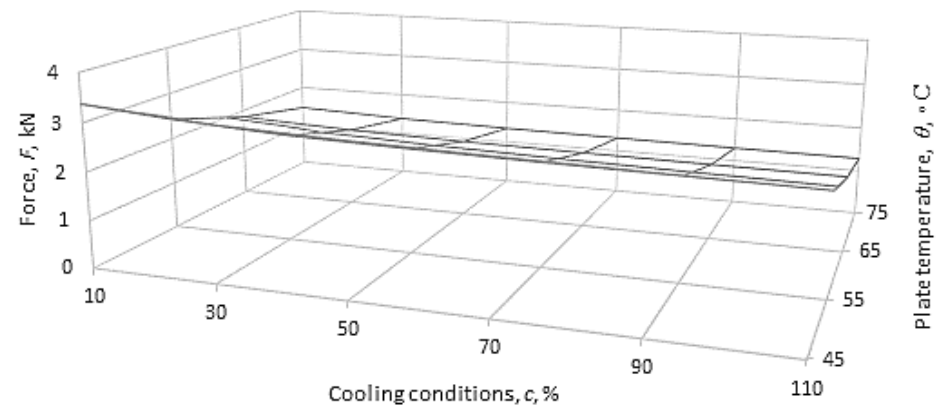

Figure 9. Effect of cooling conditions and printing plate temperature on the maximum force in the elasticity zone $(h=4 \mathrm{~mm}, v=75 \mathrm{~mm} / \mathrm{s})$

\section{Conclusions}

The use and the expected particular behavior of the plastic spherical parts under axial compression could constitute an interesting research problem. The study of the specialty literature proved that such a problem was relatively rarely approached. The investigation of the theoretical behavior of the spherical hollow parts showed that under the action of the axial compression efforts, the height of the spherical hollow parts will gradually decrease and the stress-strain curve could present a zone corresponding to the elastic deformation. When the axial force becomes higher, a plastic deformation process affects the plastic spherical hollow parts behavior. To experimentally study the behavior of the plastic hollow parts under axial compression, 9 test pieces were manufactured through a 3D printing process. Four 
manufacturing conditions were considered as process input factors, namely the part wall thickness, printing speed, cooling level, and the temperature of the printing plate. An L9 Taguchi orthogonal design with four independent variables and three experimental levels was performed. Afterward, the spherical hollow parts thus obtained were tested under axial compression efforts. By mathematical modeling of the experimental results, a power type empirical mathematical model was determined. The analysis of the model showed that the maximum influence on the elasticity of the parts was exerted by the printing plate temperature. As expected, the increase of the part wall thickness determines an increase of the plastic spherical part elasticity. In the future, extended experiments could be developed, to evaluate the effect exerted also by other manufacturing process input factors on the behavior of the parts under axial compression efforts.

Acknowlegments: This article was presented at Polymer Processing in Engineering Conference - PPE 2019, 7- 9 October 2019, Galati - Romania.

\section{References}

1. YUAN, S., CHUA, C.K., ZHOU, K.Z., Adv. Mat. Tech., 4, no. 3, 2018, 1800419.

2. KÖHNEN, P., HAASE, C., BÜLTMANN J., ZIEGLER. S., SCHLEIFENBAUM, J.H., BLECK, W., Mat. Des., 145, no. 5, 2018, p. 205.

3. YAZDIFAR, M., ESAT, I., YAZDIFAR, M., Eur. Sci. J., 14, no. 6, 2018, p. 16.

4. KOOPMAN, M. GOUADEC, G. CARLISLE, K. CHAWLA, K.-K. GLADYSZ, G., Scr. Mater., 50, no. 5,2004 , p. 593.

5. AMANI, Y., ÖCHSNER, A., Mat. -wiss. u. Werkstofftech, 46, no. 4-5, 2015, p. 462-476.

6. PILLET, M., Introduction aux plans d'expériences par la méthode Taguchi. Les Éditions d'Organisation, Paris, 1992.

7. CRETU, G., Fundamentals of experimental research. Laboratory handbook (in Romanian). "Gheorghe Asachi” Technical University of Iași, Romania, 1992.

$\overline{\text { Manuscript received: } 24.02 .2020}$ 